\title{
Limitations of Bifurcation Diagrams in Boost Converter Steady-State Response Identification
}

Preliminary Communication

\author{
Željko Stojanović \\ Zagreb University of Applied Sciences \\ Department of Electrical Engineering \\ Konavoska 2, 10000 Zagreb, Croatia \\ zeljko.stojanovic@tvz.hr
}

\author{
Denis Pelin \\ Josip Juraj Strossmayer University of Osijek \\ Faculty of Electrical Engineering, Computer Science and Information Technology Osijek \\ Kneza Trpimira 2B, 31000 Osijek, Croatia \\ denis.pelin@ferit.hr
}

\begin{abstract}
Steady-state responses of the boost converter operating in discontinuous conduction mode of operation are identified by using bifurcation diagrams as a typical simulation tool for identification of steady-state responses. The structure of simulated bifurcation diagrams is dependent on the initial period of sampling and the initial instant of sampling. The influence of these parameters on calculation of bifurcation diagrams was studied. Some possible issues, pitfalls and misinterpretations are commented as well as some recommendations about steady-state response identification are given.
\end{abstract}

Keywords - bifurcation diagram, boost converter, identification, sampling, steady-state responses

\section{INTRODUCTION}

One of the active research topics in power electronics is the analysis of possible steady-state responses of boost converters [1-4]. This is due to the widespread application of boost converter functioning as basic building blocks of a single-phase power factor correction circuit as well as executive blocks for MPPT techniques (i.e. maximum power point tracking in photovoltaic systems).

The occurrence of subharmonic, chaos and hysteretic phenomena in boost converters were studied in [5-7]. These characteristic steady-state responses are strongly dependent on the converter parameter choice. Identification of these responses in advance is of great importance for converter designers. In this way, it is possible to avoid the occurrence of some undesirable properties during service like an increased output voltage ripple or audible sound.

Typical simulation tools for identification of different steady-state responses are as follows: waveforms of state variables, their trajectories in phase plane, Poincaré maps, bifurcation diagrams and harmonic analysis [8-10].

Bifurcation diagrams are a very convenient simulation tool for steady-state response identification, because they give a qualitative insight into converter's modes of operation in a wide range of converter parameters (i.e. bifurcation parameters) [11]. The process of sampling state vari- ables, as the basic procedure for constructing bifurcation diagrams is dependent on some parameters like the initial instant of sampling in one period of an autonomous variable of the converter and the initial period of sampling. These parameters have to be properly chosen in order to avoid misinterpretation of calculated results. According to the available literature, the initial period of sampling has not yet been considered in steady-state response identification. The initial instant of sampling has been mentioned in [12] but without any detail. In typical commercial software, there are no options for setting simulation parameters like in the program written exactly for circuit analysis of the boost converter considered in this paper. That is why most researchers did not deal with this issue.

The influence of the initial period of sampling and the initial instant of sampling on the structure of bifurcation diagrams is studied in this paper. The structure of the paper is as follows. In Section 2, steady states of the boost converter are identified by measurement. Then the state equations for the mathematical model are written, whereupon the numerical mathematical method for solving state equations are applied. In Section 3, bifurcation diagrams are carried out to roughly identify steady states. Some possible issues, pitfalls and misinterpretations when choosing the initial period of sampling and the initial instant of sampling are commented. Conclusions are given in Section 4. 


\section{STEADY-STATE RESPONSES OF THE BOOST CONVERTER}

\subsection{MEASUREMENT SETUP}

The experimental boost converter is shown in Fig. 1. Steady-state responses of the boost converter are identified by varying the input voltage, i.e. the bifurcation parameter from $\mathrm{E}=15 \mathrm{~V}$ to $24 \mathrm{~V}$ with a step of input voltage $\mathrm{E}=0.1 \mathrm{~V}$, while the average value of the output voltage is set to about $25 \mathrm{~V}$.

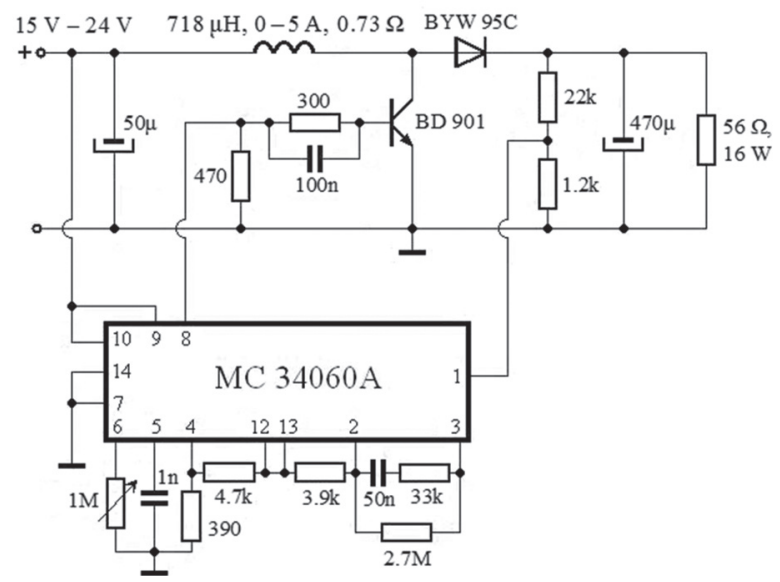

Fig. 1. Scheme of an experimental boost converter

\subsection{STEADY-STATE RESPONSES OF THE BOOST CONVERTER}

The waveforms of state variables obtained by measurement are used in steady-state response identification. The identification is done by comparing the sawtooth voltage period, $T$ and the state variable period. The waveforms of a period-2 steady state are shown in Fig 2. Steady-state responses identified by measurements for the whole range of the input voltage are shown in Table 1. A process of period doublings occurs, which is one of the known routes to chaos [13].

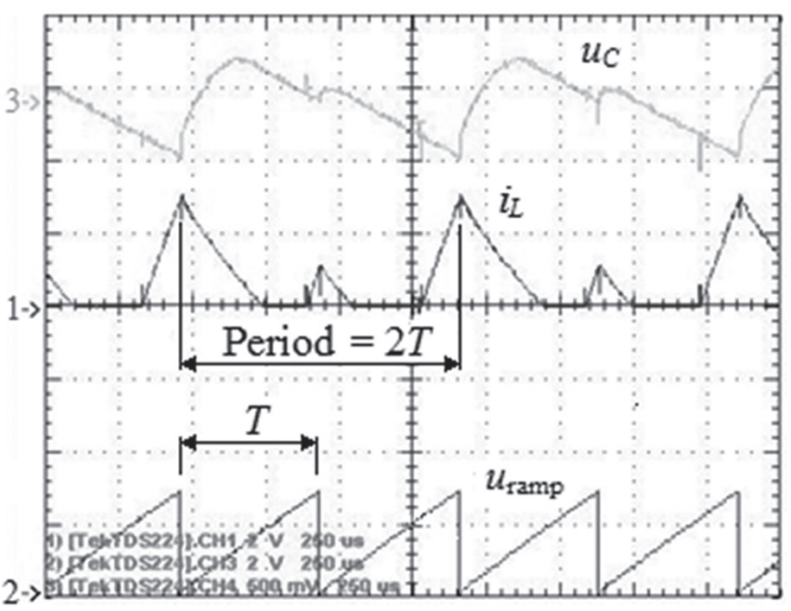

Fig. 2. Capacitor voltage, inductor current and sawtooth voltage of the period- 2 steady-state response obtained by measurement.
Table 1. Steady states obtained by measurement.

\begin{tabular}{|cc|}
\hline Steady-state responses & $\begin{array}{c}\text { Input voltage } \mathbf{E}, \mathbf{V} \\
\text { Period-1 }\end{array}$ \\
\hline Period-2 & $15.00-18.45$ \\
\hline Period-4 & $18.45-20.04$ \\
\hline Chaos & $20.04-20.84$ \\
\hline Period-3 & $20.84-22.10$ \\
\hline Chaos & $22.10-22.42$ \\
\hline
\end{tabular}

All identified steady-state responses can be displayed using one bifurcation diagram only. That is one of the reasons why bifurcation diagrams are widely used. The bifurcation diagram obtained by measurement is shown in Fig. 3. The measurement system is realized using WaveStar Software for Oscilloscopes and MATLAB and described in [14].

A mathematical model of the boost converter is derived from its scheme given in Fig. 1, and the internal schematics for the integrated circuit MC34060A, and described in more detail in [15]. It is shown in Fig. 4. It is a piecewise linear time-varying network.

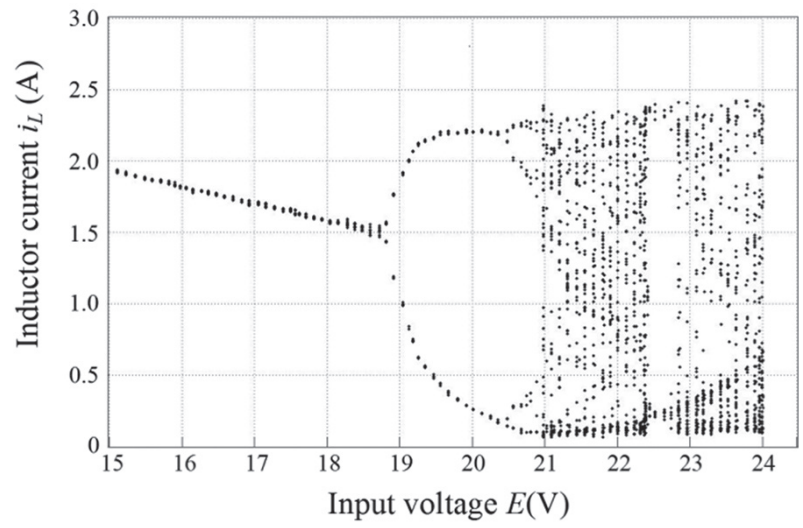

Fig. 3. Bifurcation diagram of inductor current with the input voltage as a parameter obtained by measurement, $E=15-24 \mathrm{~V}, \Delta E=0.1 \mathrm{~V}$.

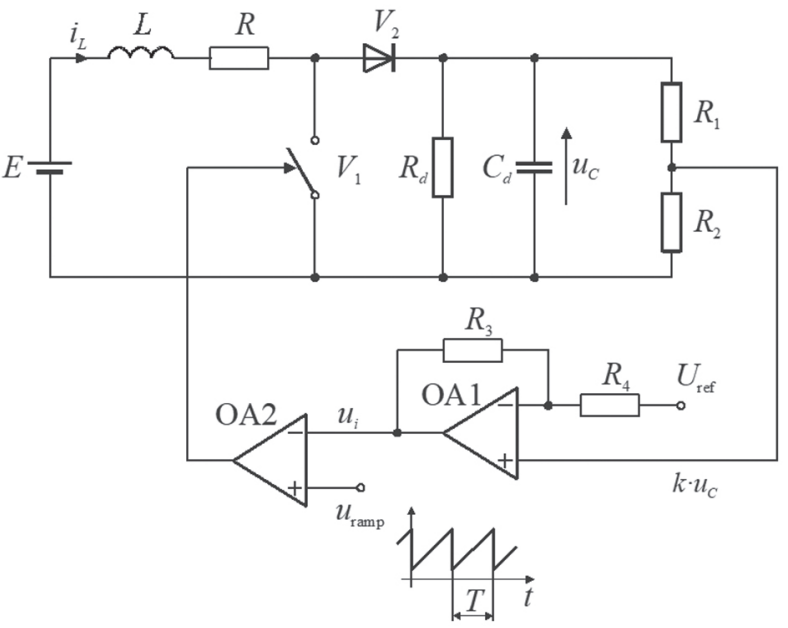

Fig. 4. The boost converter equivalent circuit. 
Assuming both continuous and discontinuous conduction mode, the converter is described by three pairs of state equations depending on the state of switches $V_{1}$ and $V_{2}$. The states of the switches are determined by the relation between voltages $u_{\mathrm{i}}$ and $u_{\text {ramp }}$ and the value of current $i_{\mathrm{L}}$ as follows:

a) When $u_{\text {ramp }} \geq u_{i}, V_{1}$ is in the ON-state and $V_{2}$ is in the OFF-state

$$
\begin{aligned}
& \frac{\mathrm{d} u_{C}}{\mathrm{~d} t}=\frac{1}{C_{d} R_{d}} u_{C} \\
& \frac{\mathrm{d} i_{L}}{\mathrm{~d} t}=\frac{1}{L}\left(E-R i_{L}\right)
\end{aligned}
$$

b) When $u_{\text {ramp }}<u_{\mathrm{i}}$ and $i_{\mathrm{L}}>0, \mathrm{~V}_{1}$ is in the OFF-state and $V_{2}$ is in the ON-state

$$
\begin{aligned}
& \frac{\mathrm{d} u_{C}}{\mathrm{~d} t}=\frac{1}{C_{d}}\left(i_{L}-\frac{u_{C}}{R_{d}}\right) \\
& \frac{\mathrm{d} i_{L}}{\mathrm{~d} t}=\frac{1}{L}\left(E-R i_{L}-u_{C}\right)
\end{aligned}
$$

c) When $u_{\text {ramp }}<u_{\mathrm{i}}$ and $i_{\mathrm{L}}=0, \mathrm{~V}_{1}$ is in the OFF-state and $V_{2}$ is in the OFF-state

$$
\begin{aligned}
& \frac{\mathrm{d} u_{C}}{\mathrm{~d} t}=\frac{1}{C_{d} R_{d}} u_{C}{ }_{\mathbb{T}} \\
& i_{L}=0
\end{aligned}
$$

The voltage

$$
u_{i}=-\frac{R_{3}}{R_{4}} U_{\text {ref }}+\left(1+\frac{R_{3}}{R_{4}}\right) \frac{R_{2}}{R_{1}+R_{2}} u_{C} \text { 的 }
$$

is determined from the equivalent circuit, and the voltage

$$
u_{\text {ramp }}=\frac{3}{T} t+0.7[\mathrm{~V}] \boldsymbol{T}
$$

is obtained by measurement and determined by the integrated circuit MC34060A.

The state equations are solved by using $4^{\text {th }}$ order Runge-Kutta method with a fixed step of integration $h=50 \mathrm{~ns}$. Model parameters are: $R_{1}=22 \mathrm{k} \Omega, R_{2}=1.22 \mathrm{k} \Omega$, $R_{3}=2.7 \mathrm{M} \Omega, R_{4}=3.9 \mathrm{k} \Omega, R=0.73 \Omega, L=698 \mu \mathrm{H}$, $C_{d}=470 \mu \mathrm{F}, R_{d}=56 \Omega, U_{\text {ref }}=5 \mathrm{~V}$ and $T=500 \mu \mathrm{s}$.

All steady-state responses identified by measurements are also observable in a simulated bifurcation diagram. The bifurcation diagram of inductor current with the input voltage as a bifurcation parameter is shown in Fig. 5. By varying the value of the input voltage $E$ in a specified range different steady-state responses are also identified by simulation according to the principle of Poincaré mapping [16]. It means that the values of a state variable, in our case the values of inductor current $i_{L}\left(t_{k}\right)$, are sampled at the instants:

$$
t_{k}=t_{0}+k T ; k=51,52, \ldots, 150,
$$

where $t_{0}$ is an instant chosen arbitrarily and $k$ is the initial period of sampling.

Each sampled point is displayed as a point on the bifurcation diagram $i_{L}\left(t_{k}\right)-E$. In this way, period - $N$ steady state of the converter will be represented by exactly $N$ points on the bifurcation diagram. Also, 100 points dispersed randomly between the maximum and the minimum value of the sampled variable $i_{L}$ indicates chaos. Bifurcation diagrams obtained by measurement and simulation correspond satisfactorily.

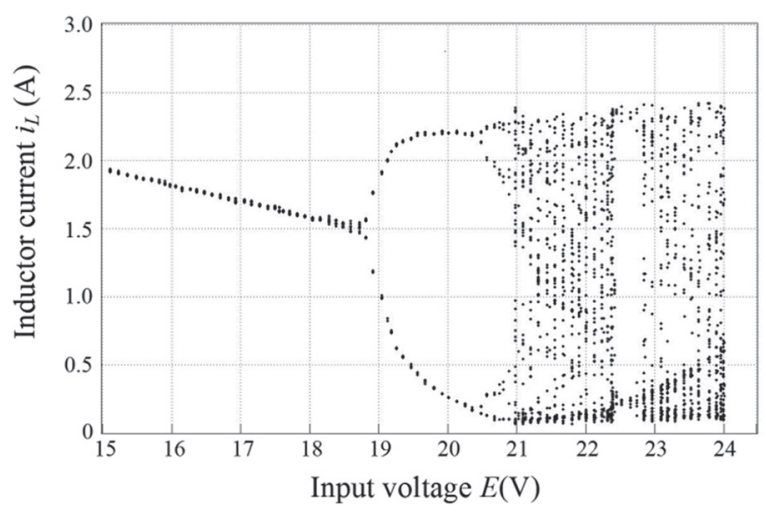

Fig. 5. Simulated bifurcation diagram of inductor current with the input voltage as a parameter. The initial instant of sampling is $t_{0}=0, E=15-24 \mathrm{~V}$, $\Delta E=5 \mathrm{mV}$ with 100 periods of sampling .

\section{LIMITATIONS OF BIFURCATION DIAGRAMS}

A few parameters of simulated bifurcation diagrams should be considered in order to get usable results: the minimal step of integration, the step of a bifurcation parameter, the initial period of sampling and the initial instant of sampling. The length of simulation to obtain a bifurcation diagram is determined by the step size of the integration method for solving state equations as well as the step of a bifurcation parameter. These two parameters were optimized with respect to the computer processor clock speed and random access memory as well as calculation time. Therefore, the fixed step size $h=50$ ns and the step of bifurcation parameter $\Delta E=5 \mathrm{mV}$ were used. In this way, 10,000 values of state variables were calculated for every sawtooth voltage period. Influences of the initial period of sampling and the initial instant of sampling are studied.

\subsection{INFLUENCE OF THE INITIAL PERIOD OF SAMPLING ON THE BIFURCATION DIAGRAM}

Change of any independent variable or change of characteristic converter's parameters will cause a change of the existing steady state through transient to a new steady state. In order to simulate bifurcation diagrams correctly, a steady state for every value of the bifurcation parameter should be accomplished.

Duration of transient depends on converter parameters as well as sawtooth voltage frequency. It is convenient to experimentally determine the duration of transient for each set of converter parameters. In our simulations, the criterion to reach a steady state is a change 
of the inductor current amplitude by less than $1 \%$. Therefore, a simulated bifurcation diagram of inductor current in the period-1 steady state as a function of the sawtooth voltage period when the initial period of sampling is set to the $50^{\text {th }}$ sawtooth voltage period is shown in Fig. 6. There are many scattered dots for any particular steady state and the range of scattered dots decreases as the sawtooth voltage period increases. One can assume that the transient between two steady states is not finished or even that the steady-state response is chaotic. Anyway, it is evident that the bifurcation diagram does not indicate the period- 1 steady state. Thereby, additional information should be acquired. The same phenomenon for the specified initial period of sampling and the sawtooth voltage period $T=50 \mu \mathrm{s}$, but now in the form of a waveform in the time domain, is shown in Fig. 7. It is evident that the transient between two steady states is not finished after 50 sawtooth voltage periods. Two periods are marked. The first one is the $50^{\text {th }}$ period, which is inappropriate as the initial period of sampling, and the second is the $260^{\text {th }}$ period, which meets the steady state criterion and is appropriate as the initial period of sampling.

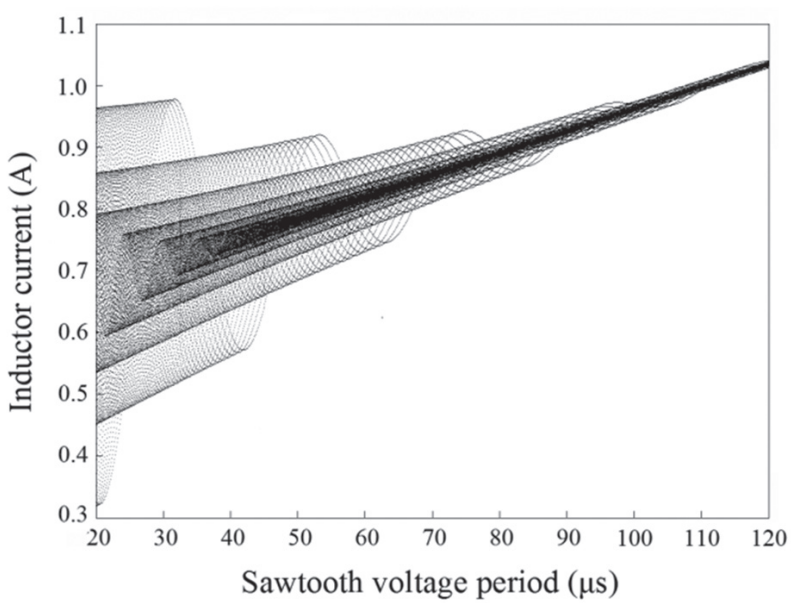

Fig. 6. Bifurcation diagram of inductor current with the sawtooth voltage period as a parameter

obtained by sampling during 100 sawtooth voltage periods starting from the 50th period after parameter change.

Therefore, for the specified sawtooth voltage period as in Fig. 7, the initial period of sampling has to be the $260^{\text {th }}$ period or more. A simulated bifurcation diagram of inductor current as a function of the sawtooth voltage period when the initial period of sampling is set to the $260^{\text {th }}$ sawtooth voltage period is shown in Fig. 8 . Period-1 can be identified when the value of the sawtooth voltage period is higher than $50 \mu \mathrm{s}$. It is also evident from Fig. 5 that the transient between two steady states in case of sawtooth voltage periods ranging between $20 \mu$ s and $50 \mu$ s is not finished even after 260 sawtooth voltage periods. In this case, the initial period of sampling has to be increased by means of the procedure shown in Figures 3 to 8. In our converter, the sawtooth voltage period is set to range between 100 $\mu \mathrm{s}$ and $500 \mu \mathrm{s}$. So, the proposed initial period $(k=50)$ of sampling is acceptable.

One has to keep in mind that the calculation time of the bifurcation diagram obtained after more sawtooth voltage periods will increase.

\subsection{INFLUENCE OF THE INITIAL INSTANT OF SAMPLING ON BIFURCATION DIAGRAMS}

Another issue when obtaining bifurcation diagrams is related to a choice of the initial instant of sampling. At first glance it looks as if it does not matter which initial instant of sampling will be chosen. Let us see the bifurcation diagrams of inductor current with the input voltage as a parameter, as in Fig. 5, but with a different initial instant of sampling. There are two bifurcation diagrams with different initial instants of sampling shown in Fig. 9. The first one is simulated for the initial instant of sampling $t 0=3 T / 5$, or for the $6,000^{\text {th }}$ value of inductor current in one period of an autonomous variable of converter, i.e. the sawtooth voltage period. The second is simulated for the initial instant of sampling t0 $=4 T / 5$. By comparing Fig. 5 and Fig. 9 it is evident that related bifurcation diagrams differ significantly although they refer to the same state variable. In Fig. 9, some branches of bifurcation diagrams are zero and it looks like they are not shown at all. That is why the information of the type of a periodic steady state is missing. The corresponding sampled values of inductor currents in Fig. 9a are marked by dots $A$ and $A^{\prime}$, while in Fig. $9 \mathrm{~b}$ they are marked by dots $B$ and $B^{\prime}$.

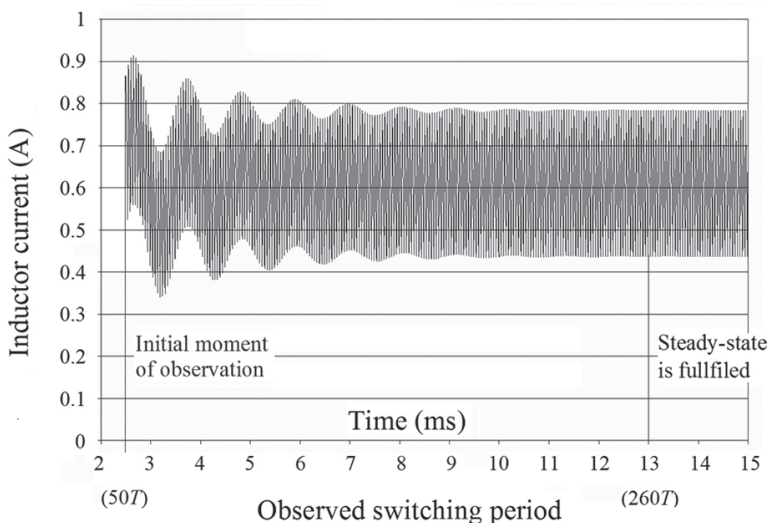

Fig. 7. Waveform of inductor current through transient to a period-1 steady state starting from the 50th period. The steady-state criterion is fulfilled at the 260th period. The sawtooth voltage period is

$$
\mathrm{T}=50 \mu \mathrm{s} \text {. }
$$

In this case, the type of a periodical steady state cannot be identified by using a bifurcation diagram of inductor current only. For example, diagrams under consideration are not convenient for identification of a steady state when the input voltage is $E=19 \mathrm{~V}$. In such case, as will be shown, other simulation tools for identification of steady-state responses have to be used. 
The Poincaré maps $u_{c}-i_{L}$ of the same simulation parameters as in Fig. 6 and the input voltage $E=19$ $V$ are shown in Fig. 10. Both Poincaré maps consist of the same number of dots. It can be concluded that the converter operates in the period- 2 steady state. This conclusion corresponds to the computed bifurcation diagram in Fig. 5. Both steady states are marked by corresponding pairs of dots $A$ and $A^{\prime}$ and $B$ and $B^{\prime}$.

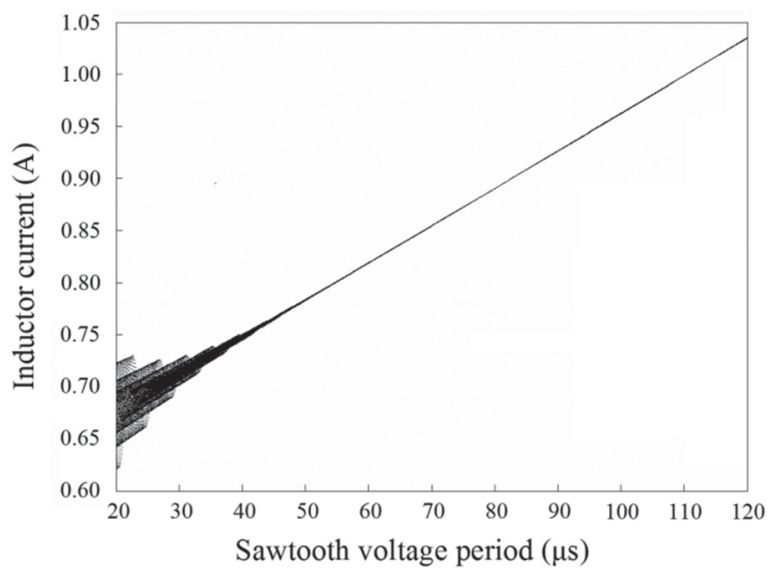

Fig. 8. Bifurcation diagram of inductor current with the sawtooth voltage period as a parameter obtained by sampling in 100 sawtooth voltage periods starting from the 260th period.
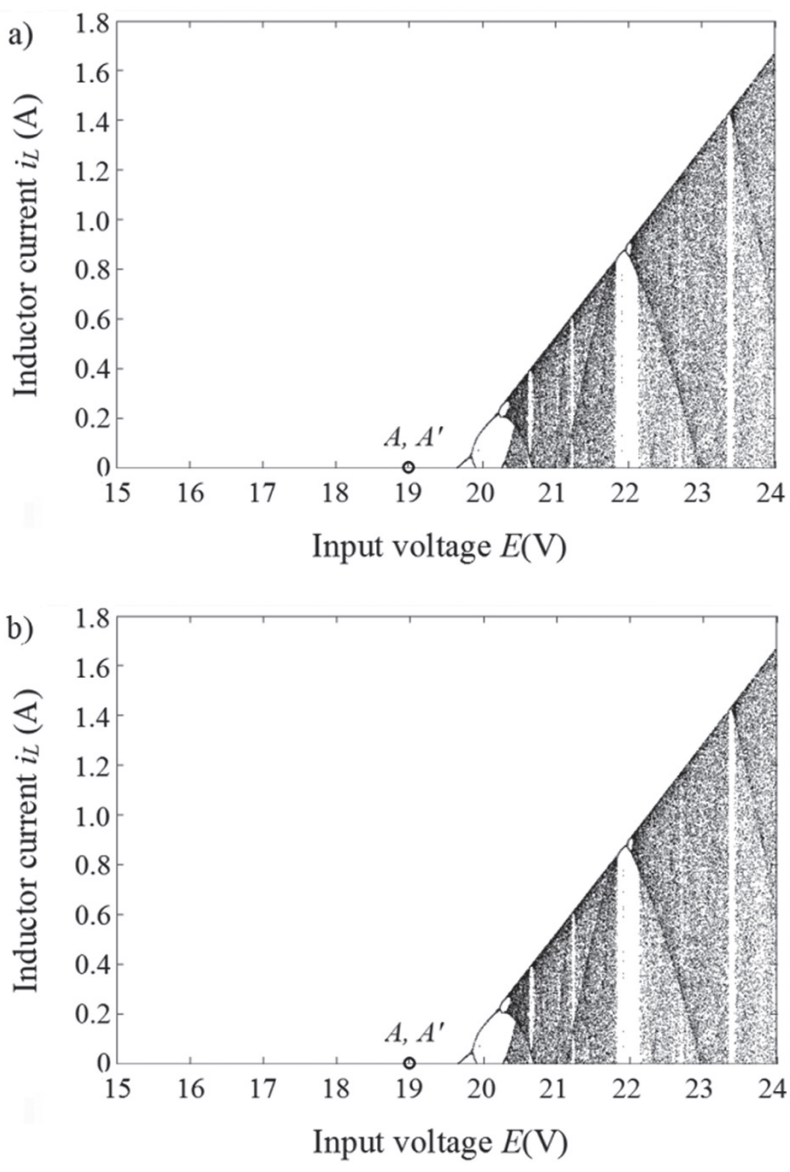

Fig. 9. Simulated bifurcation diagrams of inductor current with the input voltage as a parameter. The initial instant of sampling is: a) to $=3 \mathrm{~T} / 5, \mathrm{~b}$ ) t0 $=4 \mathrm{~T} / 5$. a)

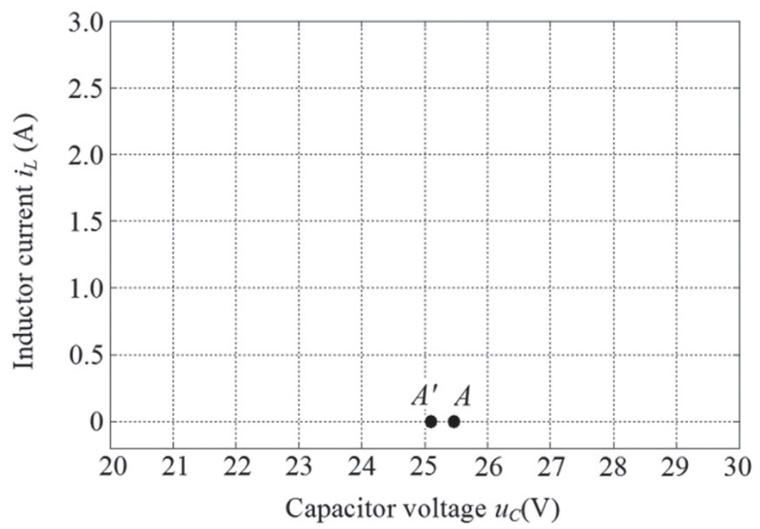

b)

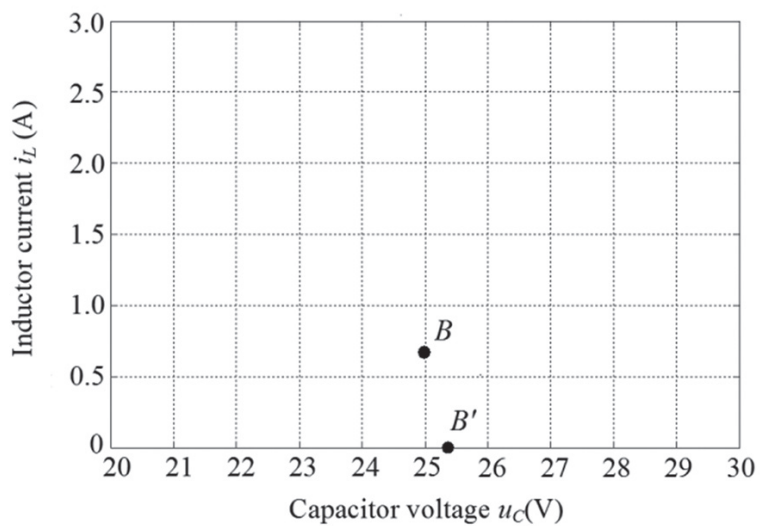

Fig. 10. Simulated Poincaré maps of inductor current with capacitor voltage. The initial instant of sampling is: a) to $=3 T / 5, b)$ to $=4 T / 5$. The input voltage is $E=19 \mathrm{~V}$.

a)

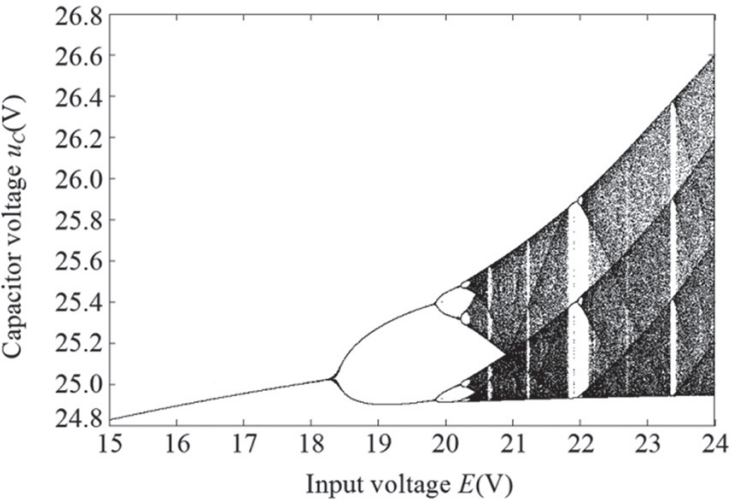

b)

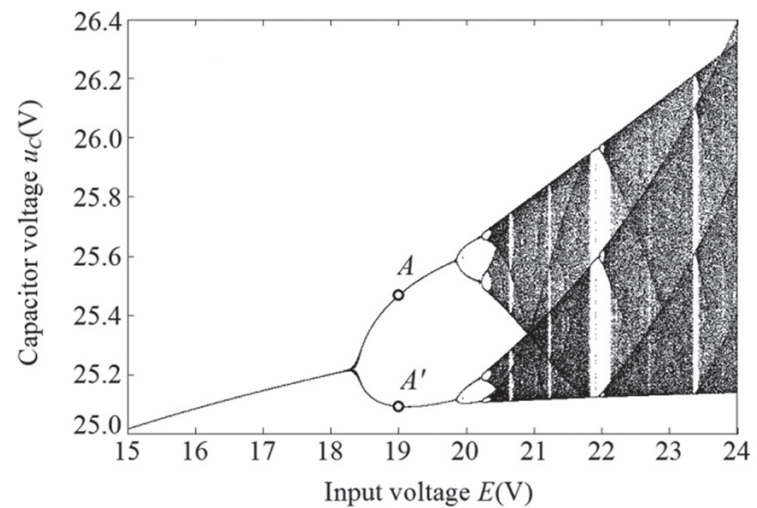


c)

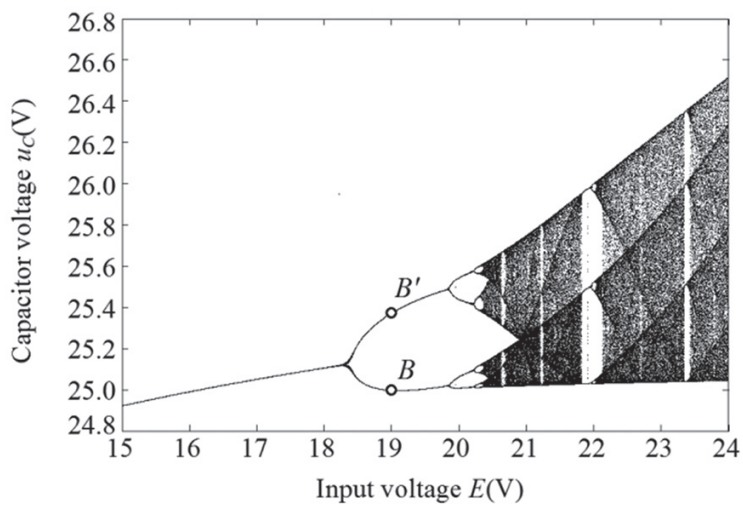

Fig. 11. Simulated bifurcation diagrams of capacitor voltage with the input voltage as a parameter. The initial instant of sampling is: a) $t_{0}={ }_{0}$, b) $t_{0}=3 T / 5$, c)

$$
t_{0}=4 T / 5 \text {. }
$$

In bifurcation diagrams in Fig. 5 and Fig. 9, some values of sampled inductor current are zero. In that case it cannot be possible to identify steady-state responses by using a bifurcation diagram only. Instead of using inductor current as a sampled variable, the other steady-state variable, i.e. output capacitor voltage, may be used. The output capacitor voltage is always greater than zero, so bifurcation diagrams will not be limited no matter of the initial instant of sampling. Bifurcation diagrams of capacitor voltage for the same initial instant of sampling as for inductor current are shown in Fig. 11.

The cross section of the plane $E=19 \mathrm{~V}$ and any of the shown bifurcation diagrams of capacitor voltage results in two dots, as marked by pairs of dots $A$ and $A^{\prime}$ and $B$ and $B^{\prime}$.

So, any of diagrams shown can be equally used for rough identification of steady-state responses of the converter. One can conclude that identification of the steady state using a bifurcation diagram of a discontinuous state variable can lead to misinterpretation when the initial instant of sampling is not properly chosen. On the other hand, the initial instant of sampling will not affect identification of the steady state of a continuous state variable as its bifurcation diagram is an invariant of the bifurcation parameter.

Nevertheless, the importance of the bifurcation diagram lies in its overall representation of steady states in a broad range of bifurcation parameters. For a reliable identification of steady-state responses of a converter operating in discontinuous conduction mode, it is recommended to use simultaneously Poincaré maps.

\section{CONCLUSIONS}

Bifurcation diagrams of the boost converter are simulated in order to roughly identify converter's steadystate responses. The influence of the initial period of sampling and the initial instant of sampling on bifurcation diagrams is studied.

It is shown that sampling of the state variable has to start after the transient is finished and a new steady state of the converter is accomplished. If the steady state is not accomplished, the bifurcation diagram will consist of many scattered dots which can lead to misinterpretation of the steady-state response. To fulfill the condition of occurrence of a new steady state, the simulation process is sometimes time consuming.

The initial instant of sampling should be taken into consideration when the converter operates in discontinuous conduction mode. In this case, the inductor current is zero during the part of the sawtooth voltage period. Thus, for some of instants of sampling one cannot identify the steady state for sure. Therefore, the bifurcation diagram of capacitor voltage has to be used simultaneously with Poincare maps in order to identify steady-state responses correctly.

\section{REFERENCES:}

[1] M. M. Al-Hindawi, A. Abusorrah, Y. Al-Turki, D. Giaouris, K. Mandal, S. Banerjee: "Nonlinear dynamics and bifurcation analysis of a boost converter for battery charging in photovoltaic applications", International Journal of Bifurcation and Chaos, Vol. 24, No. 11, 2014, 12 pp.

[2] V. Avrutin, Z. T. Zhusubaliyev, A. El Aroudi, D. Fournier-Prunaret, G. Garcia, E. Mosekilde, "Disrupted bandcount doubling in an AC-DC boost PFC circuit modeled by a time varying map", Journal of Physics: Conference Series 692, 2016.

[3] D. Pikulin, "Complete bifurcation analysis of DCDC converters under current mode control", Journal of Physics: Conference Series 482, 2014.

[4] Ž. Stojanović, I. Božić, M. Miletić, "Boost converter steady-state dependence on switching frequency", Polytechnic\&Design, Vol. 4, No. 3, 2016, pp. 232-237.

[5] S. Banerjee, K. Chakrabarty, "Nonlinear modelling and bifurcations in the boost converter", IEEE Transactions on Power Electronics, Vol.13, No. 3, 1998, pp. 252-260.

[6] A. El Aroudi, R. Leyva, "Quasi-Periodic Route to Chaos in a PWM Voltage-Controlled DC-DC Boost Converter", IEEE Transactions on Circuits and Systems I: Fundamental Theory and Applications, Vol. 48, No. 8, 2001, pp. 967-978.

[7] Ž. Stojanović, D. Pelin, K. Predrijevac, "Problem of obtaining boot converter bifurcation model for hysteretic behaviour", Proceedings of the $17^{\text {th }}$ International Conference on Electrical Drives and 
Power Electronics, Dubrovnik, Croatia, 2-4 October 2013, pp. 94-98.

[8] C. K. Tse, "Complex behaviour of switching power converters", CRC Press, 2004.

[9] D. Pelin, I. Flegar, K. Miličević, "Identification of steady-state responses of the boost converter", Technical Gazette, Vol.16, No. 2, 2009, pp. 9-15.

[10] K. T. Chau, Z. Wang, "Chaos in electric drive systems: analysis, control and application", Wiley-IEEE Press, 2011.

[11] I. Flegar, D. Pelin, "Bifurcation diagrams - Insight into the modes of operation of a boost converter", Proceedings of the IEEE International Conference on Industrial Technology, Maribor, Slovenia,10-12 December 2003, pp. 836-841.

[12] B. Basak, S. Parui, "Incompleteness of bifurcation diagram in predicting the behavior of Cuk converter in discontinuous conduction mode", Inter- national Journal of Circuit Theory and Applications, Vol. 36, No. 4, 2007, pp. 387-396.

[13] C. K. Tse, "Flip Bifurcation and Chaos in Three-State Boost Switching Regulators", IEEE Transactions on Circuits and Systems I: Fundamental Theory and Applications, Vol. 41, No. 1, 1994, pp. 16-23.

[14] D. Pelin, I. Biondic, I. Brnjevarac, "Experimental bifurcation diagrams of a non-isolated DC-DC converters", Proceedings of the $18^{\text {th }}$ International Symposium on Power Electronics, Novi Sad, Serbia, 28-30 October 2015, pp. 1-5.

[15] D. Pelin, K. Miličević, "Parameter space maps of the boost converter", Electronics, Vol. 11, No. 1-2, 2007, pp. 43-47.

[16] A. El Aroudi, M. Debbat, L. Martínez-Salamero, "Poincaré maps modeling and local orbital stability analysis of discontinuous piecewise affine periodically driven systems", Nonlinear Dynamics, Vol. 50, No. 3, 2007, pp. 431-445. 